http://dx.doi.org/10.21707/gs.v11.n03a07

\title{
EsTUdos DO SISTEMA CLIMA URBANO: LEVANTAMENTO BIBLIOGRÁFICO de dissertações do Prodema e sua relaÇão Com a Temática nos ÚTIMOS 16 ANOS
}

\author{
Marcus Raffael Oliveira da Silva ${ }^{1 *}$, Joel Silva dos Santos ${ }^{2}$
}

${ }^{1}$ Graduado em Ecologia pela Universidade Federal da Paraíba, Campus IV - Litoral Norte - Rio Tinto, PB - Brasil.

${ }^{2}$ Docente Adjunto do Departamento de Engenharia e Meio Ambiente, (UFPB $\backslash$ Campus IV) e do Programa de Pós-Graduação em Desenvolvimento e Meio Ambiente $\backslash U F P B$.

*Autor para correspondência: marcusraffael@outlook.com

Recebido em 08 de setembro de 2016. Aceito em 19 de junho de 2017. Publicado em 29 de julho de 2017.

REsumo - O presente estudo trata de um levantamento de natureza bibliográfica sobre os trabalhos referentes ao estudo da climatologia urbana na Rede do Programa de Pós-Graduação em Desenvolvimento e Meio Ambiente -PRODEMA nos últimos 16 anos. A coleta dos dados ocorreu por meio de consultas aos bancos de dados dos programas de Pós-Graduação de diversas universidades que integram a Rede PRODEMA em nível de Mestrado. Os campos de investigação que definem o Sistema Clima Urbano de Monteiro (1976) - Campo Termodinâmico; Físico-Químico e Hidrometeorórico - serviram como estratégia de classificação para análise da produção científica a respeito da temática. Os resultados da pesquisa demonstram que foram encontradas um total de 1000 dissertações de mestrado que versavam sobre diversas temáticas da área das ciências ambientais no período de 2000 a 2016 (Fevereiro) na Rede Prodema. No entanto, foram encontradas apenas 15 dissertações de mestrado sobre o tema clima urbano, dessas 14 enfatizaram o campo termodinâmico e apenas um trabalho tratou de avaliar o campo hidrometeórico.

Palavras-Chave: Sistema Clima Urbano; Campo Térmico; UrbanizaÇÃo.

System urban Climatestudy: Bibliographical search of Prodema dissertations and its relation to the theme FOR THE LAST 16 YEARS

Aвstract - This study is a survey on bibliographic data of works related to urban climate studies performed by the Development and Environment Post-Graduation Program - PRODEMA over the last 16 years. Data collection occurred by consulting the PRODEMA data banks in several universities at Master's level. The investigation fields that define the Monteiro's Urban Climate System (1976) - Thermodynamic, Physical-Chemical and Hidrometeoricfields - worked as a classifying strategy for the scientific production analysisregarding the theme. The survey results show that, inside the PRODEMA network, there was 1,000 dissertations regarding many areas inside environmental sciences from 2000 to 2016 (February). However, 15 dissertations over the urban climatesubjectwere found, in which 14 emphasized the thermodynamic field and only one study attempted to assess the hidrometeoric field.

Keywords: URBAN CLIMATE SYSTEM; THERMODYNAMIC FIELD; URBANIZATION.

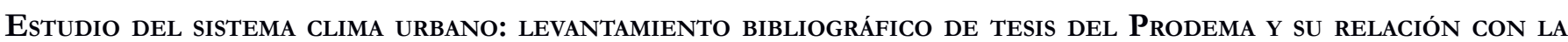
TEMÁTICA EN LOS ÚlTIMOS 16 AÑos

RESUMEN - El presente estudiotrata de un levantamiento denaturaleza bibliográficasobrelos trabajos referentes al estudio de la climatología urbana en la Red del Programa de Posgrado en Desarrollo y Medio Ambiente - PRODEMA-enlos 
últimos16 años. La recopilación de datos ocurrió por medio de consultas a las bases de datos de los programas de posgrado de diversas universidades que integran la Red PRODEMA en el nivel de Maestría. Los campos de investigación que definen el Sistema Clima Urbano de Monteiro (1976) - Campo Termodinámico; Físico-químico e Hidrometereológico - sirvieron como estrategia de clasificación para análisis de la producción científica sobre el tema. Los resultados dela investigación demuestran que se encontraro nun total de 1.000 tesis de Maestría que trataban sobre diversas temáticas del área de las ciencias ambientales en el período de 2000 a 2016 (febrero) en la Red PRODEMA. Sin embargo, sólo se encontraron 15 tesis de maestría sobre el tema clima urbano, de estas 14 enfatizaron el campo termodinámico y apenas un trabajo trató de evaluarel campo hidrometereológico.

Palabras Clave: Sistema Clima Urbano; Campo térmico; Urbanización.

\section{INTRODUÇÃO}

A Revolução Industrial acarretou diversas transformações no espaço geográfico, afetando diversos sistemas ambientais, e especificamente, o sistema climático das cidades. Verifica-se que a partir do processo de industrialização, e consequentemente, de urbanização acelerada, as cidades sofreram grandes impactos ambientais que afetaram a qualidade de vidas da população residente nesses ambientes. Várias transformações ocorreram nos padrões da vida humana e consequentemente na mudança física do espaço natural, destaca Mills et al. (2010).

Santos (2011) adverte que nas últimas décadas o sistema atmosférico urbano é um dos mais alterados pela mudança do uso e cobertura do solo urbano, redução das áreas verdes, ações antropogênicas diversas, adensamento urbano etc.

Segundo Basane et al. (2011), mais de 80\% da população mundial reside em áreas urbanas alterando a paisagem natural, especialmente em países em desenvolvimento. Para Carfan (2011), o aumento da população e a modificação na paisagem, alteram o clima local, gerando um clima específico das cidades: clima urbano. Para ambos os autores, conhecer o clima urbano, fazer um planejamento adequado e entender suas delimitações e consequências, é de grande interesse para uma melhor qualidade de vida e o planejamento ambiental de áreas urbanas.

De acordo com Basane et al. (2011), é notório que a urbanização e a industrialização juntas afetam as caraterísticas da atmosfera e alteram os elementos climáticos: temperatura do ar, umidade relativa, vento, radiação solar e dentre outros. A urbanização acelera o crescimento das estruturas urbanas, tanto verticalmente como horizontalmente, o que consequentemente, altera a direção dos ventos, a temperatura do ar, a umidade relativa, absorção da água e a evapotranspiração em áreas urbanas. Ainda em relação à industrialização e seu impacto no sistema atmosférico, Leal et al. (2008), afirmam que o clima é afetado através da construção de grandes empreendimentos de engenharia, usinas hidrelétricas, termoelétricas e termonucleares, pela exploração mineral e pela construção de ferrovias e rodovias (sem analisar os impactos causados pela agricultura, pecuária, silvicultura, caça e pesca).

Para Monteiro e Medonça (2003), o clima urbano é um sistema que envolve o clima de um determinado espaço terrestre e sua urbanização. Segundo Monteiro (1976), o clima urbano pode ser dividido em três subsistemas de análise: termodinâmico, físico-químico e o hidrometeórico.

De acordo com Monteiro (1976), o clima urbano de cada cidade tem condições climáticas próprias. O mesmo autor afirma que as consequências da formação do clima urbano são diretas, acarretando às populações 
o desconforto térmico, a poluição do ar, os impactos pluviais e a formação das ilhas de calor.

O mesmo autor também destaca que com o crescimento da urbanização, a qualidade de vida de uma parte da população melhorou, o que acarretou em outros problemas ambientais com o aumento da fabricação e frota de veículos para uma maior mobilidade nas cidades e a aclimatização de ambientes individualizados. Dessa forma pode-se inferir que o crescimento da urbanização é um dos fatores que influencia fortemente a degradação ambiental. A impermeabilização dos solos e a redução das áreas verdes nos centros urbanos tem alterado o sistema clima urbano e gerado diversos problemas socioambientais para as cidades.

É diante deste contexto que este artigo se insere, procurando identificar a quantidade de dissertações defendidas na Rede PRODEMA sobre a temática em questão nos últimos 16 anos.

O PRODEMA é constituído de uma rede regional de Programas de Pós-Graduação em Desenvolvimento e Meio Ambiente, que reúne atualmente sete IES (Instituto de Ensino Superior) públicas nordestinas de quase todos os estados da região. O PRODEMA oferta cursos em nível de mestrado e doutorado em desenvolvimento e meio ambiente, e se constitui em um Programa de Pós-Graduação de caráter interdisciplinar.

Atualmente a rede de programas do PRODEMA é composta por 07 Universidades:Universidade Federal da Paraíba - UFPB, Universidade Federal de Pernambuco - UFPE, a Universidade Federal de Sergipe - UFS, Universidade Federal do Piauí - UFPI, a Universidade Federal Rio Grande do Norte - UFRN, Universidade Federal do Ceará - UFC e a Universidade Estadual Santa Cruz - UESC (Ilhéus-BA).

Dessa forma o objetivo central deste artigo é apresentar a distribuição espacial e temporal dos estudos de clima urbano desenvolvidos na rede de programas do PRODEMA em nível de Mestrado, destacando os campos de investigação do Sistema Clima Urbano proposto por Monteiro (1976) que define em três subsistemas interligados: termodinâmico, físico-químico e hidrometeórico, além dos autores e cidades onde os trabalhos foram desenvolvidos.

\section{Metodologia}

Para a realização do trabalho, inicialmente foi feito um levantamento bibliográfico a respeito da temática em geral, e realizada a consulta nos sites e no Sistema Integrado de Gestão de Atividades Acadêmicas - SIGAA de cada instituição que compõe o PRODEMA. Em seguida, após abusca no banco de dados de Dissertações de cada Programa de Pós-Graduação em Desenvolvimentoe Meio Ambiente que integra a Rede PRODEMA, foi realizada a classificação e quantificação dos trabalhos desenvolvidos no PRODEMA no período compreendido entre 2000 e 2016. As pesquisas forma classificadas segundo o enfoque do Sistema Climático Urbano de Monteiro (1976) que define três campos de estudo do clima urbano: o termodinâmico, físico-químico e hidrometeórico. Também foi realizada a categorização por autor, ano e a cidade onde foi desenvolvida a pesquisa.

Vale ressaltar, que o fato da Universidade Federal de Alagoas (UFAL), da Universidade Estadual do Rio Grande do Norte (UERN) e da Universidade Estadual da Paraíba (UEPB) terem se desvinculado da Rede PRODEMA, essas não foram citadas nesta pesquisa, uma vez que as mesmas, não disponibilizam mais, seu banco de dados de dissertações de Mestrado em seus sites.

\section{Resultados E Discussão}


Segundo o PRODEMA UFPB (2016), a criação inicial dos programas que compõe a Rede PRODEMA, surgiu no final da década de 1980 com a iniciativa da Universidade Federal de Alagoas (UFAL). Posteriormente foi apresentado um projeto de Pós-Graduação em rede durante a 54 Reunião Plenária do Conselho de Reitores das Universidades Brasileiras, realizada em março de 1992, na Universidade Federal de Sergipe (UFS). O projeto foi aprovado e assinado por dezoito Instituições Universitárias Nordestinas, onde em seu Protocolo de Intenções foi criadoo PRODEMA.

Depois da criação da rede do PRODEMA, logo em seguida as universidades foram criando resoluções que davam direito e autorizavam a implementação do curso em nível de mestrado (Tabela 1).

Tabela 1 - Universidades que compõe a rede de programas do PRODEMA em nível de Mestrado e seu respectivo ano de criação.

\begin{tabular}{cc}
\hline Programas & $\begin{array}{c}\text { Ano de Criação } \\
\text { do Mestrado }\end{array}$ \\
\hline UFPB & \\
UEPB & 1995 \\
UFAL & \\
UEPB & \\
UFS & \\
UFC & \\
UFPE & 1996 \\
& 1998 \\
UESC & 2002 \\
UFPI & 2003 \\
\hline UFRN & \\
\hline
\end{tabular}

Conforme observado na Tabela 1 foram apresentados os Programa de Pós- Graduação da UFPB, UEPB, UFAL, UERN, UFS e UFC como os mais antigos, ou seja, pioneiros a fazer parte da Rede PRODEMA em nível de Mestrado, sendo que a UEPB, a UFAL e a UERN se desvincularam da Rede PRODEMA. Em seguida os demais programas, a UFPE (1996), a UESC (1998), a UFPI (2002) e a UFRN (2003).

A pesquisa realizada na base de dados da rede de programas que integram o PRODEMA encontrou um total de 1000 dissertações de mestrado que versam sobre diversos temas dentro da área de ciências ambientais no período compreendido entre o ano 2000 e 2016. Desse total, apenas 15 dissertações de mestrado abordaram o tema clima urbano.

$\mathrm{Na}$ Figura 1, são apresentadosos dados referentes às produções das Universidades que compõem a Rede PRODEMA. 
Figura 1 - Dissertações de Mestrado definidas no período de 2000 a 2016.

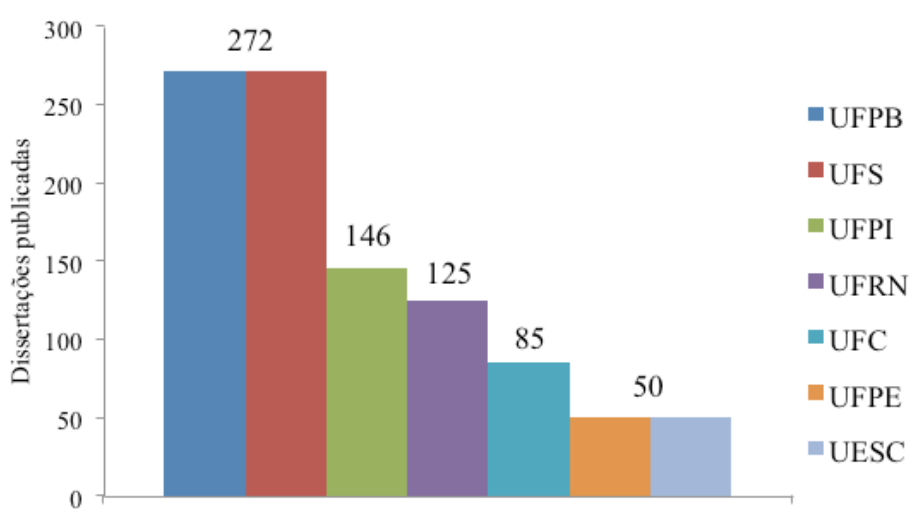

Fonte: Banco de dados darede de programas do PRODEMA.

Conforme observado na Figura 1, fica evidente que as universidades que mais defenderam dissertações no período de 2000 a 2016 foram a UFPB e a UFS. Cada uma delas apresentou 272 dissertações concluídas. A UFPI apresentou 146 dissertações; a UFRN apresentou 125 dissertações; a UFC apresentou 85 dissertações. Já a UFPE e a UESC apresentaram cada uma 50 dissertações defendidas.

De acordo com o banco de dados de dissertações da Rede PRODEMA, a primeira dissertação defendida sobre a temática do clima urbano foi a de Silva (2000), enfatizando o conforto térmico ambiental nas edificações de Mangabeira VII, João Pessoa, PB. Esse trabalho foi realizado no PRODEMA \UFPB. Devido a notoriedade do tema das mudanças climáticas globais nos últimos anos, verificou-se um o crescente interesse pelo tema em estudo. Isso pode ser observado recentemente com a conclusão de três dissertações versando sobre a temática no PRODEMA \UFPB: Freitas (2015); Aranha (2016) e Vieira (2016).

A Tabela 02 apresenta o quantitativo total de dissertações de mestrado que foram defendidas na Rede PRODEMA ao longo do período investigado. No Programa de Pós- Graduação da UFPB, uma das universidades pioneiras da Rede PRODEMA, verificou-se as dissertações sobre a temática clima urbano dos seguintes autores: Silva (2000), Medeiros (2001), Ramos (2002), Furtado (2002), Lima (2004), Coutinho (2004), Costa (2008), Freitas (2015), Vieira (2016) e Aranha (2016). Todos os trabalhos são referentes às condições térmicas e/ou o conforto humano, ou seja, ao campo termodinâmico. Com relação aos demais sistemas de análise não foram encontrados dissertações defendidas no período estudado.

As pesquisas de Freitas (2015), Vieira (2016) e Aranha (2016) usaram metodologias adaptadas a partir de Monteiro (1976); Katzschner (2002); Costa (2007) e Santos (2011), em que os autores avaliaram o clima urbano através de descrições físicas do espaço ocupado aliadas às medições in loco e a análise das variáveis climáticas de temperatura e umidade relativa do ar.

Para a UFPI, os estudos na área da climatologia urbana se concentraram no campo térmico. Os trabalhos foram desenvolvidos por: Aguilera (2006), Santos (2010), Feitosa (2010) e Albuquerque (2012), predominando os estudos de clima urbano com ênfase nas edificações e conforto térmico dos bairros da cidade de Teresina, Piauí.

Na UFRN, foi encontrado 01 estudo. A pesquisa realizada por Costa (2010) merece destaque, pois foi à única da Rede PRODEMA a estudar o campo hidrometeórico,

Para UFPE, UFS, UESC e UFC nenhum trabalho foi mencionado sobre os subsistemas climáticos em 
nível de dissertação de mestrado.

$\mathrm{Na}$ Tabela 2 são apresentados os dados referentes ao numero de dissertações publicadas sobre o clima urbano nas Universidades que compõem a Rede PRODEMA.

Tabela 2 - Quantificação dos estudos de clima urbano na rede de programas do PRODEMA em nível de Mestrado, no período de 2000 a 2016.

\begin{tabular}{|c|c|c|c|c|c|}
\hline \multirow{2}{*}{ Programa } & \multirow{2}{*}{$\begin{array}{l}\text { Cidade onde } \\
\text { a pesquisa foi } \\
\text { realizada }\end{array}$} & \multirow{2}{*}{ Autor/Ano } & \multicolumn{3}{|c|}{ Subsistemas ou Canais de Percepção do S.C.U. } \\
\hline & & & $\begin{array}{c}\text { Termodinâmico } \\
\text { (Conforto Térmico) }\end{array}$ & $\begin{array}{l}\text { Físico-Químico } \\
\text { (Qualidade do Ar) }\end{array}$ & $\begin{array}{l}\text { Hidrometeórico (Im- } \\
\text { pacto Meteórico) }\end{array}$ \\
\hline \multirow{10}{*}{ UFPB } & & Silva (2000) & & & \\
\hline & & Medeiros (2001) & & & \\
\hline & & Ramos (2002) & & & \\
\hline & Natal & Furtado (2002) & & & \\
\hline & João Pessoa & Lima (2004) & & & \\
\hline & $\begin{array}{c}\text { Campina Gran- } \\
\text { de }\end{array}$ & Coutinho (2004) & 10 & - & - \\
\hline & Patos & Costa (2008) & & & \\
\hline & & Freitas (2015) & & & \\
\hline & & Vieira (2016) & & & \\
\hline & & Aranha (2016) & & & \\
\hline \multirow{4}{*}{ UFPI } & & Aguilera (2006) & & & \\
\hline & & Santos (2010) & & & \\
\hline & Teresina & Feitosa $(2010)$ & 04 & - & - \\
\hline & & Albuquerque (2012) & & & \\
\hline UFRN & Pau dos Ferros & Costa $(2010)$ & - & - & 01 \\
\hline UFPE & - & - & - & - & - \\
\hline UFS & - & & - & - & - \\
\hline \multirow[t]{2}{*}{ UFC } & - & - & - & - & - \\
\hline & & Total & 14 & 0 & 01 \\
\hline
\end{tabular}

Conforme observado na Tabela 2 encontra-se o quantitativo de estudos realizados por cidades, autores/ anos, em relação às três áreas de aplicação do S.C.U. proposta por Monteiro (1976). Apesar de muitos trabalhos analisados aqui não adotar a metodologia de Monteiro (1976) os mesmos foram classificados nos níveis de 
abordagem que formam a estrutura do S.C.U.

Comparando-se os resultados obtidos no presente estudo como os de Moura e Zanella (2013) que investigaram os trabalhos referente a temática clima urbanos nos Programas de Pós-Graduação em Geografia, observa-seo predomínio de estudos sob o enfoque do campo térmico (total de 68 estudos), seguido dos campos hidrometeórico (08 estudos) e físico-químico (04 estudos). Em seus estudos, as cidades que mais tiveram trabalhos sobre o clima urbano foi Fortaleza e Recife que apresentaram estudos nos três campos do S.C.U, seguido de Natal e Salvador que além de possuírem pesquisas no campo térmico, apresentaram estudos aplicados, de modo respectivo, nos campos físico-químico e hidrometeórico. Uma possível explicação para o fato de haver uma predominância no campo termodinâmico entre os estudos pode estar relacionado ao clima quente e úmido da região Nordeste que gera ilhas de calor e desconforto térmico em várias cidades dessa região.

\section{Conclusão}

Foi encontrado um total de 1000 dissertações de mestrado que versam sobre diversas temáticas da área das ciências ambientais no período de 2000 a 2016 (Fevereiro) na Rede PRODEMA. Através do estudo realizado foi possível destacar os Programas de Pós- Graduação daUFPB e a UFS com o maior número de dissertações defendidas, totalizando 272 dissertações cada uma.

Baseando-se nos resultados obtidos pode-se inferir que os estudos relacionados ao clima urbano estão vinculados principalmente ao campo termodinâmico e foram desenvolvidos principalmente nos Programas de Pós- Graduação da UFPB (10 trabalhos) e a UFPI (04 trabalhos). Foi encontrado apenas um estudo no campo hidrometeórico do Sistema Climático Urbano proposto por Monteiro (1976). Esse trabalho foi realizado pelo PRODEMA \UFRN. Com relação ao campo Físico-Químico nenhum trabalho foi mencionado durante o período investigado, ou seja, nos últimos dezesseis anos.

No artigo também ficou perceptível à falta de atualização dos bancos de dados de algumas Universidades que compõem a Rede PRODEMA (UFPE, UFS, UFPI, UFRN, UFC e UESC) dificultando assim, o acesso às informações necessárias para este estudo.

Portanto, o presente estudo fornecerá subsídios para a elaboração depesquisas com temas diversos relacionados ao levantamento bibliográfico das dissertações do PRODEMA referente às Ciências Ambientais, e especialmente à temática do Clima Urbano.

\section{REFERÊNCIAS}

Aguilera RCAO. 2006. Conforto térmico e coberturas: Estudos para o aumento da eficiência energética em edificações. Piauí: UFPI. Dissertação de Mestrado.

Albuquerque MM. 2012. Relação entre uso e ocupação do solo e variáveis climáticas: Estudo em bairros da cidade de Teresina, Piauí. Piauí: UFPI. Dissertação de Mestrado.

Aranha KC. 2016. Tecnologias sustentáveis: a importância dos telhados verdes na eficiência energética e amenização microclimática. João Pessoa: UFPB. Dissertação de Mestrado. 
Basane AC, Silva RF, Miranda YC, Baldo MC. 2011. I Simpósio de Estudos Urbanos: Desenvolvimento Regional e Dinâmico Ambiental. ISSN: 2236-4056.

Carfan AC. 2011. Analise do conforto térmico em áreas abertas no município de Ourinhos - SP. São Paulo: Faculdade de Filosofia, Letras e Ciências Humanas da Universidade de São Paulo. 168p. Tese de Doutorado.

Costa ADLO. 2007. Revestimento de superfícies horizontais e sua implicação microclimática em localidade de baixa latitude com clima quente e úmido. Campinas: UNICAMP, 242p. Tese Doutorado.

Costa FR. 2010. Inundações urbanas no semiarido Nordestino: O caso da cidade de Pau Dos Ferros RN. Rio Grande do Norte: UFRN. Dissertação de Mestrado.

Costa SC. 2008. Percepção e uso de diretrizes de sustentabilidade na elaboração de projetos arquuitetônicos na cidade de Natal-RN. João Pessoa: UFPB. Dissertação de Mestrado.

Coutinho MAF. 2004. Evolução Urbana e Qualidade de Vida: O caso da Avenida Epitácio Pessoa. João Pessoa: UFPB. Dissertação de Mestrado.

Feitosa SMR. 2010. Alterações climáticas em Tteresina - PI decorrentes da urbanização e supressão de áreas verdes. Piauí: UFPI. Dissertação de Mestrado.

Freitas AF. 2015. Clima urbano eplanejamento ambiental: Um estudo de caso no espaço intra-urbano do campus I da UFPB. João Pessoa: UFPB. Dissertação de Mestrado.

Furtado RNO. 2002. A Segregação Residencial em J Pessoa-PB: Impactos na Qualidade de Vida e Ambiental (Estudo sobre a ocupação do Loteamento Jardim da Cidade Universitária). João Pessoa: UFPB. Dissertação de Mestrado.

Katzschner L, Bosch U, Rottgen M. 2002. Behaviour of people in open spaces in dependency of thermal comfort conditions. In: Internacional Conference on Passive And Low Energy ARCHITECTURE PLEA, 19, France.

Leal GCSG, Farias MSS, Araujo AF. 2008. O processo de industrialização e seus impactos no meio ambiente urbano. Qualitas Revista Eletrônica. 7(1). INSS: 1677-4280.

Lima MAS. 2004. Morfologia Urbana, Qualidade de Vida e Ambiental em Assentamentos Espontâneos: O caso do bairro São José - João Pessoa - PB. João Pessoa: UFPB. Dissertação de Mestrado.

Medeiros MAS. 2001. Verdes Urbanos: uma análise da contribuição da vegetação ao conforto ambiental na cidade de Patos - PB. João Pessoa: UFPB. Dissertação de Mestrado.

Mills G, Cleugh H, Emmanuel R, Endlicher W, Erell E, Mcgranahan G, Ng, E, Nickson A,Rosenthal J, Steemer K. 2010. Climate Information for Improved Planning and Management of Mega Cities (Needs Perspective). Procedia Environmental Sciences, 1(1): 228-246.

Monteiro CA de F. 1976. Teoria e clima urbano. Série Teses e Monografias, São Paulo:USP/Igeog, n 25. 
Monteiro CAF, Mendonça F. 2003. Clima Urbano. In: Inês MorescoDanni- Oliveira, Ana Maria De Paiva Macedo Brandão, Neyde Maria Santos Gonçalves, (Colaboradores).- São Paulo: Contexto.

PRODEMA - UESC. 2016. Prodema UESC. Disponível em: http://www.uesc.br/cursos/pos_graduacao/ mestrado/mdrma/index.php?item=conteudo dissertacoes.php. Acesso em: 03 Agos 2016.

PRODEMA - UFC. 2016. Prodema UFC. Disponível em: http://www.prodema.ufc.br/ Acesso em: 03 Agos 2016.

PRODEMA - UFPB. 2016. Histórico do Prodema. Disponível em: http://www.prpg.ufpb.br/prodema/ novosite/prodema.php. Acesso em: 01 Agos 2016.

PRODEMA - UFPE. 2016. Prodema UFPE. Disponível em: https://www.ufpe.br/prodema/. Acesso em: 01 Agos 2016.

PRODEMA - UFPI. 2016. Prodema UFPI. Disponível em: http://www.leg.ufpi.br/mestambiente/index/ pagina/id/2528. Acesso em: 03Agos 2016.

Ramos MMQ. 2002. O clima no planejamento de Campina Grande - PB: dinâmica urbana e aumento da temperatura. . João Pessoa: UFPB. Dissertação de Mestrado.

Santos FAC. 2010. Desempenho térmico de edificações multipavimentos: Análise do uso de fachadas de vidro diretamente expostas à radiação solar na cidade Teresina - PI. Piauí: UFPI. Dissertação de Mestrado.

Santos JS. 2011. Campo térmico urbano e a sua relação com uso e cobertura do solo em uma cidade tropical úmida. Campina Grande: UFCG. 108 p. Tese de Doutorado.

SIGAA - Sistema Integrado de Gestão de Atividades Acadêmicas (UFC). 2016. Programa de Teses e Dissertações da UFC. Disponível em: https://si3.ufc.br/sigaa/public/programa/defesas.jsf?lc=pt BReid=10014. Acesso em: 03 Agos 2016.

SIGAA - Sistema Integrado de Gestão de Atividades Acadêmicas (UFPB). 2016. Programa de Teses e Dissertações da UFPB. Disponível em: http://www.prpg.ufpb.br/prodema/novosite/prodema.php. Acesso em: 01 Agos 2016.

SIGAA - Sistema Integrado de Gestão de Atividades Acadêmicas (UFPI). 2016. Programa de Teses e Dissertações da UFPI. Disponível em: https://www.sigaa.ufpi.br/sigaa/public/programa/portal.jsf?id=619. Acesso em: 03Agos 2016.

SIGAA - Sistema Integrado de Gestão de Atividades Acadêmicas (UFRN). 2016. Programa de Teses e Dissertações da UFRN. Disponível em: https://sigaa.ufrn.br/sigaa/public/programa/defesas.jsf?lc=pt BReid=423. Acesso em: 03 Agos 2016.

SIGAA - Sistema Integrado de Gestão de Atividades Acadêmicas (UFS). 2016. Programa de Teses e Dissertações da UFS. Disponível em: https://www.sigaa.ufs.br/sigaa/public/programa/portal.jsf?id=135. 
Acesso em: 01 Agos 2016.

Silva LFG. 2000. Análise de fatores determinantes de conforto térmico Ambiental em conjuntos habitacional de baixa renda: O Caso dos apartamentos de Mangabeira VII, João Pessoa - PB. João Pessoa: UFPB. Dissertação de Mestrado.

Vieira DS. 2016. Internações por doenças do aparelho circulatório em idosos e suas relações com o campo térmico urbano de duas cidades Paraibanas. João Pessoa: UFPB. Dissertação de Mestrado.

Zanella ME, Moura MO. 2013. O clima das cidades do Nordeste Brasileiro: Contribuições no Planejamento e Gestão Urbana. Revista da ANPEGE. 9(11):.75-89.ISSN: 1679-768 X. 